\title{
Reliability of direct genomic values for animals with different relationships within and to the reference population
}

\author{
M. Pszczola, ${ }^{\star} \dagger \ddagger^{1}$ T. Strabel, $\neq$ H. A. Mulder, ${ }^{\star}$ and M. P. L. Calus ${ }^{\star}$ \\ *Animal Breeding and Genomics Centre, Wageningen UR Livestock Research, 8200 AB Lelystad, the Netherlands \\ †Animal Breeding and Genomics Centre, Wageningen University, $6700 \mathrm{AH}$ Wageningen, the Netherlands \\ ‡Department of Genetics and Animal Breeding, Poznan University of Life Sciences, Wolynska 33, 60-637 Poznan, Poland
}

\begin{abstract}
Accuracy of genomic selection depends on the accuracy of prediction of single nucleotide polymorphism effects and the proportion of genetic variance explained by markers. Design of the reference population with respect to its family structure may influence the accuracy of genomic selection. The objective of this study was to investigate the effect of various relationship levels within the reference population and different level of relationship of evaluated animals to the reference population on the reliability of direct genomic breeding values (DGV). The DGV reliabilities, expressed as squared correlation between estimated and true breeding value, were calculated for evaluated animals at 3 heritability levels. To emulate a trait that is difficult or expensive to measure, such as methane emission, reference populations were kept small and consisted of females with own performance records. A population reflecting a dairy cattle population structure was simulated. Four chosen reference populations consisted of all females available in the first genotyped generation. They consisted of highly (HR), moderately (MR), or lowly (LR) related animals, by selecting paternal half-sib families of decreasing size, or consisted of randomly chosen animals (RND). Of those 4 reference populations, RND had the lowest average relationship. Three sets of evaluated animals were chosen from 3 consecutive generations of genotyped animals, starting from the same generation as the reference population. Reliabilities of DGV predictions were calculated deterministically using selection index theory. The randomly chosen reference population had the lowest average relationship within the reference population. Average reliabilities increased when average relationship within the reference population decreased and the highest average reliabilities were achieved for RND (e.g., from 0.53 in $\mathrm{HR}$ to 0.61 in RND for a heritability of 0.30 ). A higher relationship
\end{abstract}

Received March 4, 2011.

Accepted September 18, 2011.

${ }^{1}$ Corresponding author: mbee@jay.up.poznan.pl to the reference population resulted in higher reliability values. At the average squared relationship of evaluated animals to the reference population of 0.005 , reliabilities were, on average, 0.49 (HR) and 0.63 (RND) for a heritability of $0.30 ; 0.20(\mathrm{HR})$ and 0.27 (RND) for a heritability of 0.05 ; and 0.07 (HR) and 0.09 (RND) for a heritability of 0.01 . Substantial decrease in the reliability was observed when the number of generations to the reference population increased [e.g., for heritability of 0.30 , the decrease from evaluated set I (chosen from the same generation as the reference population) to II (one generation younger than the reference population) was 0.04 for HR, and 0.07 for RND]. In this study, the importance of the design of a reference population consisting of cows was shown and optimal designs of the reference population for genomic prediction were suggested.

Key words: genomic selection, reference population design, reliability, direct genomic value

\section{INTRODUCTION}

Practical applications of genomic selection (GS; Meuwissen et al., 2001) are becoming more popular in animal (Hayes et al., 2009b) and plant breeding (Heffner et al., 2009; Jannink et al., 2010). Several countries use genomic information as one of the sources of information for selection in breeding programs. Genomic selection uses genome-wide SNP markers. Due to high marker density, all QTL are assumed to be in linkage disequilibrium (LD) with markers on the SNP chip (Meuwissen et al., 2001). This implies that a single SNP marker or a group of markers can be associated with QTL effects (Grapes et al., 2004, 2006; Yu et al., $2005)$. Based on those associations, genotypic information can be used as an additional source of information to increase the reliability of EBV, thereby increasing the accuracy of selection.

The accuracy of GS depends on 2 factors (Daetwyler et al., 2008; Goddard, 2009): (1) the accuracy of estimated SNP effects and (2) the proportion of the genetic variance explained by the markers. The accuracy of 
estimated SNP effects is influenced by the size of the reference population; that is, the number of animals with genotypes and phenotypic records used to estimate SNP effects and the heritability of the considered trait. The proportion of genetic variance explained by the markers is influenced by the effective size of the considered population $\left(\boldsymbol{N}_{\boldsymbol{e}}\right)$ and the density at which the SNP chip covers the genome. The effective population size influences the proportion of genetic variance explained by the markers. At low $N_{e}$, the number of independent segments present in the genome is expected to be lower (Goddard, 2009). Fewer independent segments implies that fewer markers are needed to tag all segments and fewer records are needed to estimate effects of these segments (Goddard, 2010). Therefore, the accuracy of GS is expected to be higher in a population with smaller $N_{e}$ than in a population with large $N_{e}$.

The reliability of direct genomic values (DGV) increases together with an increase of the reference population size, as the accuracy of estimating SNP effects increases. This was shown in theoretical studies (Goddard, 2009; Meuwissen, 2009), simulation studies (Meuwissen et al., 2001; Pszczola et al., 2011), and real data analysis (Lund et al., 2010; for reviews, see Hayes et al., 2009b; Calus, 2010). Furthermore, it was suggested that use of a reference population comprising animals with a wide range of phenotypes and genotypes would yield reliable predictions across the range of genotypes included in the reference population (Calus, 2010). In addition, the family structure of the reference population may influence the reliability of GS, as shown by Pérez-Cabal et al. (2010).

The information content of phenotypes of animals in the reference population depends on their accuracy; for example, their correlation with the true breeding value of the animal. For example, in dairy cattle, bulls are usually included in the reference population using their daughter yield deviations (Gonzalez-Recio et al., 2008) or deregressed breeding values that are estimated with high reliability (Berry et al., 2009; Schenkel et al., 2009; VanRaden et al., 2009). This in turn requires numerous measurements on close relatives for those animals. Routinely recorded traits may easily meet this requirement; however, for traits difficult or expensive to measure, the number of observations may be limited and therefore daughter yield deviations (DYD) or deregressed EBV may not be available. Considering, for example, methane emission, where measuring a single observation is much more expensive than the genotyping costs per animal, it may be cost inefficient to phenotype many daughters per sire. To balance genotyping and phenotyping costs in such cases, we expect that genotyping cows that are phenotyped would be more efficient than genotyping only bulls and aggregating cow phenotypes at the bull level. In such a scenario, however, the reference population will consist of a limited number of animals with phenotypes that have lower heritability than DYD or deregressed EBV. When the reference population is small and the heritability of phenotypes is low, the reliability of predictions based on estimated SNP effects is expected to be low as well. The design of such a small reference population may be important therefore to maximize the reliability of predictions.

When considering an evaluated animal, a close relationship to the animals included in the reference population is expected to give a more reliable prediction (Habier et al., 2007; Legarra et al., 2008; Sonesson and Meuwissen, 2009). Optimally, all evaluated animals should have at least some closely related animals in the reference population. These observations suggest that the design of the reference population, in terms of relationships within and to the reference population, has to be considered. The design of the reference population may be especially important when the reference population consists of a limited number of individuals. With a small reference population, the reliability of DGV is expected to be low and any increase in the reliability is desired. To date, only a few results have been reported on the effect of the relationships within the reference population on the reliability of DGV for evaluated animals (Pérez-Cabal et al., 2010).

The objective of this study was to investigate the effect of various relationship levels within the reference population and the level of relationship of evaluated animals to the reference population on the reliability of DGV. The reliabilities of DGV, expressed as squared correlation between estimated and true breeding value, were calculated for the evaluated animals at 3 levels of heritability. To represent a dairy cattle breeding program aiming to initiate a reference population for a new trait, which is difficult or expensive to measure, animals included in the reference population were females and assumed to have only their own performance records. The size of the reference population was therefore assumed to be small.

\section{MATERIALS AND METHODS}

In this study, a dairy cattle population structure was simulated. Four reference populations with different family structures were selected. Three sets of evaluated animals with different numbers of generations to the chosen reference populations were sampled from simulated animals that were not included in 1 of the 4 reference populations. The evaluated animals were genotyped but had no phenotypes. The animals chosen for the reference populations and evaluated animals were all females. Reliabilities of DGV predictions for 
the evaluated animals were calculated and compared for 3 different heritability levels $(0.30,0.05$, and 0.01$)$.

\section{Reliabilities of DGV Calculation}

Reliabilities of DGV predictions for all evaluated animals were calculated deterministically for 2 scenarios, without predicting the DGV themselves: (1) reliability based on pedigree relationships of the animals and their phenotypes $\left(r_{A}^{2}\right)$; and (2) reliability based on the approach in which pedigree relationships of the animals were replaced by genomic relationships $\left(r_{G}^{2}\right)$.

Formulas to calculate $r_{A}^{2}$ and $r_{G}^{2}$ can be derived from selection index theory (see Appendix) or from the prediction error variances of the mixed model equations used to estimate the breeding values. Values of $r_{A}^{2}$ were calculated for evaluated animals as

$$
r_{A}^{2}=\mathbf{a}\left[\mathbf{A}+\mathbf{I}\left(\frac{\sigma_{e}^{2}}{\sigma_{A}^{2}}\right)\right]^{-1} \mathbf{a}^{\prime},
$$

where $\mathbf{a}$ is a vector with pedigree based relationships of an evaluated animal with the animals in the reference population; $\mathbf{A}$ is the additive relationship matrix for animals in the reference population; $\mathbf{I}$ is an identity matrix, $\sigma_{e}^{2}$ is the residual variance; and $\sigma_{A}^{2}$ is the genetic variance. The $\sigma_{e}^{2} / \sigma_{A}^{2}$ ratio reflects the heritability $\left(h^{2}\right)$.

Calculation of $r_{G}^{2}$ was similar to that in [1] but used genomic relationship coefficients instead of pedigreebased relationships (VanRaden, 2008):

$$
r_{G}^{2}=\mathbf{c}\left[\mathbf{G}+\mathbf{I}\left(\frac{\sigma_{e}^{2}}{\sigma_{A}^{2}}\right)\right]^{-1} \mathbf{c}^{\prime},
$$

where $\mathbf{c}$ is a vector with genomic relationships of an evaluated animal with the animals in the reference population; $\mathbf{G}$ is the genomic relationship matrix for animals in the reference population. $\mathbf{G}$ is constructed as $\frac{\mathbf{Z Z}^{\prime}}{2 \sum p_{i}\left(1-p_{i}\right)}$, following VanRaden (2008), where $p_{i}$ is the frequency of the second allele at locus $i$, and $\mathbf{Z}$ is derived from genotypes of animals in the reference population, by subtracting 2 times the allele frequency expressed as a difference of 0.5 , that is $2\left(p_{i}-0.5\right)$, from matrix $\mathbf{M}$ that specifies the marker genotypes for each individual as $-1,0$, or 1 , and vector $\mathbf{c}$ is a column of the $\mathbf{C}$ matrix for a particular evaluated animal. The $\mathbf{C}$ matrix is created as $\frac{\mathbf{Z}_{2} \mathbf{Z}^{\prime}}{2 \sum p_{i}\left(1-p_{i}\right)}$, where $\mathbf{Z}_{2}$ is constructed from genotypes of evaluated animals (VanRaden, 2008).

\section{Simulation}

The simulated genome was $3 \mathrm{M}$ long and consisted of 3 chromosomes with a length of $1 \mathrm{M}$ each. This corresponds, approximately, to $10 \%$ of the cattle genome (Ihara et al., 2004). In the first generation, monomorphic marker loci $(300,000)$ were spaced at fixed distances of $0.001 \mathrm{cM}$ across the genome.

de Roos et al. (2009) presented a simulation scheme mimicking different sizes of $N_{e}$ at different stages in the historic cattle population, using inflated values for the frequency of mutation events and recombination rates. This simulation scheme was adopted in the present study. For the first phase, an historical population of 100 individuals was simulated and randomly mated for 600 generations; the frequency of mutation events was $10^{-4}$ per locus per generation and the recombination rate was $10^{4}$ per M per generation. Subsequently, 200 generations were simulated, using a frequency of mutation events per locus per generation of $10^{-7}$ and a recombination rate per $M$ per generation of 100 . In the third phase, the number of simulated individuals was increased to 10,000 cows and 100 sires. To mimic the third phase described by de Roos et al. (2009), each sire was mated to 100 randomly chosen dams for the next 50 generations. The recombination rate was set to 1 per $\mathrm{M}$ per generation and mutations were stopped. In the last phase, the number of sires was reduced to 25 and the number of randomly chosen dams per sire increased to 400 . This last phase was repeated for 15 generations to mimic the phase of modern cattle breed. Thereafter, another 5 generations were simulated in which the number of sires was increased to 50 and the number of offspring was, on average, 200 per sire. Pedigree was stored for the 10 last generations and genotypes for the 3 last generations. The simulation was performed according to the outline presented in Table 1 with parameters summarized in Table 2 . Twenty replicates were simulated for each scenario and heritability combination.

\section{Scenarios}

Three sets of evaluated animals were chosen from the 3 consecutive generations of the genotyped animals. The evaluated set I and the reference populations were chosen from the same generation. The evaluated sets II and III were, respectively, 1 and 2 generations further away from the reference populations. Each set of animals consisted of 1,000 individuals originating from only one generation at the time. The evaluated sets consisted of animals sired by 50 sires. The 20 offspring per sire were chosen randomly from all available offspring of that sire. 
Table 1. Simulation outline: number of generations and animals, mutation and recombination rates, mimicked number of generations, and simulated effective population size $\left(N_{e}\right)^{1}$

\begin{tabular}{lrrccrr}
\hline $\begin{array}{l}\text { No. of } \\
\text { generations }\end{array}$ & $\begin{array}{c}\text { No. of } \\
\text { sires }\end{array}$ & $\begin{array}{c}\text { No. of } \\
\text { females }\end{array}$ & $\begin{array}{c}\text { Mutation rate } \\
\text { (per locus) }\end{array}$ & $\begin{array}{c}\text { Recombination rate } \\
\text { (per M/gen) }\end{array}$ & $\begin{array}{c}\text { Mimicked no. } \\
\text { of generations }\end{array}$ & $\begin{array}{c}\text { Simulated } \\
N_{e}\end{array}$ \\
\hline 600 & 50 & 50 & $10^{-4}$ & $10^{4}$ & $6,000,000$ & $\sim 1,000,000$ \\
200 & 50 & 50 & $10^{-7}$ & 100 & 20,000 & $\sim 12,500$ \\
50 & 100 & 10,000 & No mutation & 1 & 50 & $\sim 400$ \\
15 & 25 & 10,000 & No mutation & 1 & 15 & $\sim 100$ \\
5 & 50 & 10,000 & No mutation & 1 & 5 & $\sim 200$ \\
\hline
\end{tabular}

${ }^{1}$ Simulation outline adopted from de Roos et al. (2009).

Reference populations were chosen from all females that had not been chosen in the evaluated set. All the reference populations consisted of 2,000 cows. The chosen reference populations differed by their family structure. The first reference population (HR) consisted of highly related animals; the second (MR) consisted of moderately related animals; the third $(\mathbf{L R})$ consisted of lowly related animals; and the fourth (RND) consisted of randomly selected animals. Differences in the level of the relationship within the reference populations were achieved by choosing paternal half-sib families of different sizes in each of the 4 scenarios. For $\mathrm{HR}$, sires with at least 425 offspring were selected and from them offspring of 5 randomly chosen sires were included. For MR, offspring of 20 randomly chosen sires were included. For LR, offspring of 40 randomly chosen sires were included. For RND, 2,000 randomly chosen animals were included. In HR, MR, and LR, each sire contributed with equal number of offspring (400, 100, and 50, respectively) chosen randomly from all their available offspring. Reliabilities of DGV were calculated for each of the 3 sets of evaluated animals with use of the 4 different reference populations, using equations [1] and [2].

\section{RESULTS}

\section{Characteristics of the Simulations}

In each replicate, animals with genotypes were simulated. The average distance between adjacent segregating loci across the whole genome, calculated in the last generation, was approximately $0.03 \mathrm{cM}$ (Table 2 ). The average LD between adjacent markers, measured as $\mathrm{r}^{2}$ (Hill and Robertson, 1968), was 0.23, and minor allele frequency averaged across all marker loci in the last generation was 0.25 . The calculated allele frequencies were consistent across the reference populations. Among all the chosen reference populations, RND had the lowest average relationship.

The reference population, composed of progeny of only 5 sires (HR) on average, resulted in an average pedigree relationship of about 0.09 . The average relationship in HR was higher than that in MR by about 0.04; differences in the average relationship levels among MR, LR, and RND were small (0.06 to 0.05) as shown in Table 3.

\section{Reliabilities of Predictions}

Use of Genomic Information. The increase in the reliabilities was clearly higher when the heritability was higher, as illustrated in Figures 1 and 2. The level of increase caused by using genomic information was similar across all reference populations (Figure 1). The increase in reliability caused by using genomic information, averaged across all the reference populations and evaluated sets, was $0.39,0.13$, and 0.03 , respectively, for the highest, medium, and lowest heritabilities. Figure 3 shows that reliabilities increased when pedigree information was replaced by genomic information. The individual reliability values varied. For HR, 2 distinct groups of reliability values were found: high and low. The many more reliability values in the low group compared with the high group led to a lower mean value of reliability across individuals for HR compared with

Table 2. Simulation parameters

\begin{tabular}{|c|c|c|c|}
\hline Parameter & \multicolumn{3}{|c|}{ Value } \\
\hline Simulated genome length $(\mathrm{M})$ & \multicolumn{3}{|c|}{3} \\
\hline Number of simulated chromosomes & \multicolumn{3}{|c|}{3} \\
\hline Length of simulated chromosomes (M) & \multirow{2}{*}{\multicolumn{3}{|c|}{$\stackrel{1}{\sim 003}$}} \\
\hline Distance between adjacent markers in the last generation (cM) & & & \\
\hline Number of SNP markers per cM & \multicolumn{3}{|c|}{$\sim 39.1$} \\
\hline Minor allele frequency & \multicolumn{3}{|c|}{0.25} \\
\hline & Trait 1 & Trait 2 & Trait 3 \\
\hline Heritability & 0.30 & 0.05 & 0.01 \\
\hline
\end{tabular}


Table 3. Average pedigree-based relationship within the reference population, across different scenarios averaged over all replicates

\begin{tabular}{llc}
\hline & \multicolumn{2}{c}{ Relationship } \\
\cline { 2 - 3 } Scenario $^{1}$ & Mean & SD \\
\hline HR & 0.0946 & 0.0048 \\
MR & 0.0562 & 0.0020 \\
LR & 0.0497 & 0.0016 \\
RND & 0.0487 & 0.0016 \\
\hline
\end{tabular}

${ }^{1} \mathrm{HR}=$ the reference population with the highest average relationship within the reference population; $\mathrm{MR}=$ the reference population with moderate average relationship within the reference population; LR $=$ the reference population with the lowest average relationship within the reference population; RND = the reference population consisted of randomly selected individuals.

RND. In RND, no distinct groups of reliabilities were present and variance of reliabilities was much smaller.

Family Structure of the Reference Population. The reliabilities increased when the average relationship within the reference population decreased (e.g., from 0.477 in $\mathrm{HR}$ to 0.597 in RND for heritability of 0.30) as illustrated in Figures 1 and 2 and Table 4. The family structure of the reference population, therefore, strongly influenced the average relationship of the reference population and therefore had an effect on reliabilities.

Relationship to the Reference Population. Three different measures of the relationship level of the evaluated animals to the reference population were calculated: average relationship, average squared relationship, and the maximum relationship. To determine which of these measures was most closely related to the reliability, the individual reliabilities of the animals included in the evaluated set I were compared with each of those measures, as shown in Figure 4. The reliabilities calculated with pedigree and genomic information were the most closely related to average squared relationship. The reliabilities showed a poorer relationship to the 2 other measures. The average squared relationship, therefore, is used hereafter as a measure of the relationship to the reference population.

Figure 2 shows comparison of the reliabilities for HR and RND at different levels of the average squared relationship to the reference population. It can be seen that when the half-sib families in the reference population were smaller (RND), the reliabilities at the given level of the average squared relationship to the reference population were higher. Reliabilities were regressed on the average squared relationships to the reference population (Figure 2). Regression coefficients increased with heritability level and were considerably higher for RND than for HR. This dependency was consistent over all the replicates and was observed across all the heritabil- ity levels, as well as for all evaluated sets of animals (results not shown). Animals with a higher average squared relationship to the reference population (based on genomic information) also had higher values of the reliability. This trend was observable across all reference populations and heritability levels. For example, at the average squared relationship of 0.005 , the reliability for the heritability of 0.30 was, on average, 0.49 for HR and 0.63 for RND. For the lower heritability levels, the differences between HR and RND were smaller: 0.20 for HR and 0.27 for RND at a heritability of 0.05 , and 0.07 for HR and 0.09 for RND at a heritability of 0.01 . Some variation in the reliability level at certain levels of the average squared relationship was found. This variation was smaller for the reference population with smaller families, as shown in Figure 2.

Increasing the number of generations to the chosen reference populations resulted in a decrease in reliability (Figure 1). This decrease in the reliability was substantial when the predictions were based on pedigree relationships. The individual reliabilities obtained for the evaluated animals from different generations and their levels of the average squared relationship to the reference population were compared (results not shown). This comparison revealed that the decrease in the reliability was mostly explained by a reduction in the average squared relationship to the reference population. The decrease tended to be larger when the average relationship within the reference population was smaller. For example, for a heritability of 0.30 , the decrease in reliability due to increased number of generations in the reference population was 0.042 for HR and 0.109 for RND (from the evaluated set I to II), and 0.067 for HR and 0.167 for RND (from the evaluated set I to III; see Figure 1A and Table 4). For the remaining heritability levels, this decrease in reliability was somewhat lower, but still substantial (Figure 1). The decrease in reliability caused by the increased number of generations between the reference population and the evaluated animals was smaller with use of genomic data than pedigree data. For example, for heritability of 0.30 , the decrease in reliability caused by an increased number of generations in the reference population was 0.035 for HR, 0.066 for RND from the evaluated set I to II and 0.061 for HR and 0.108 for RND from the evaluated set I to III (Figure 1B and Table 4). The level of reliability, moreover, decreased more slowly over the generations when genomic data were used (Figure 1). When using genomic data, for a heritability of 0.30 , the decrease in reliability tended to be higher when the average relationship within the reference population decreased (Table 4). Although the decrease was larger for $\mathrm{RND}$, the ranking of the scenarios remained the same. 
a)
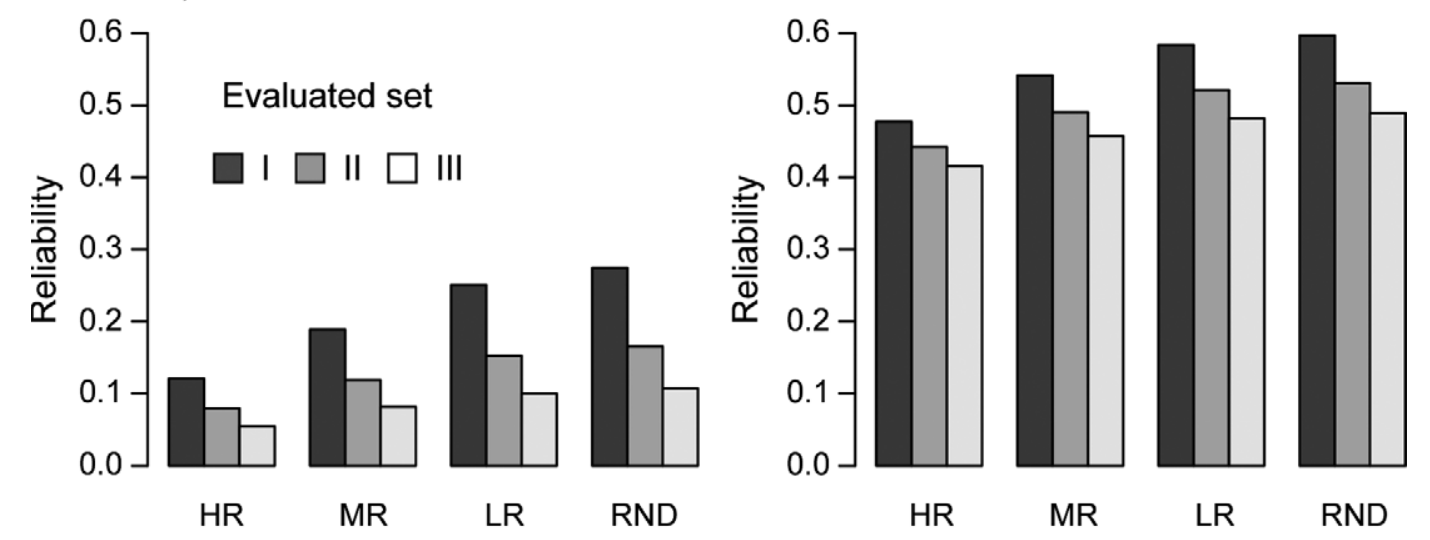

b)
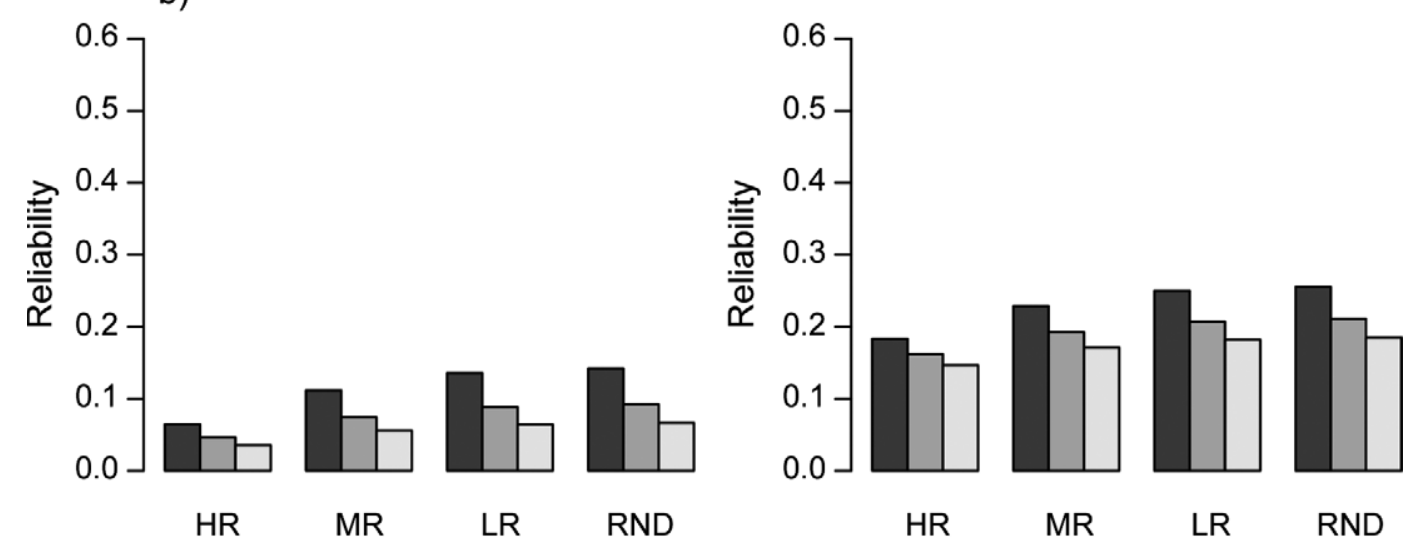

c)
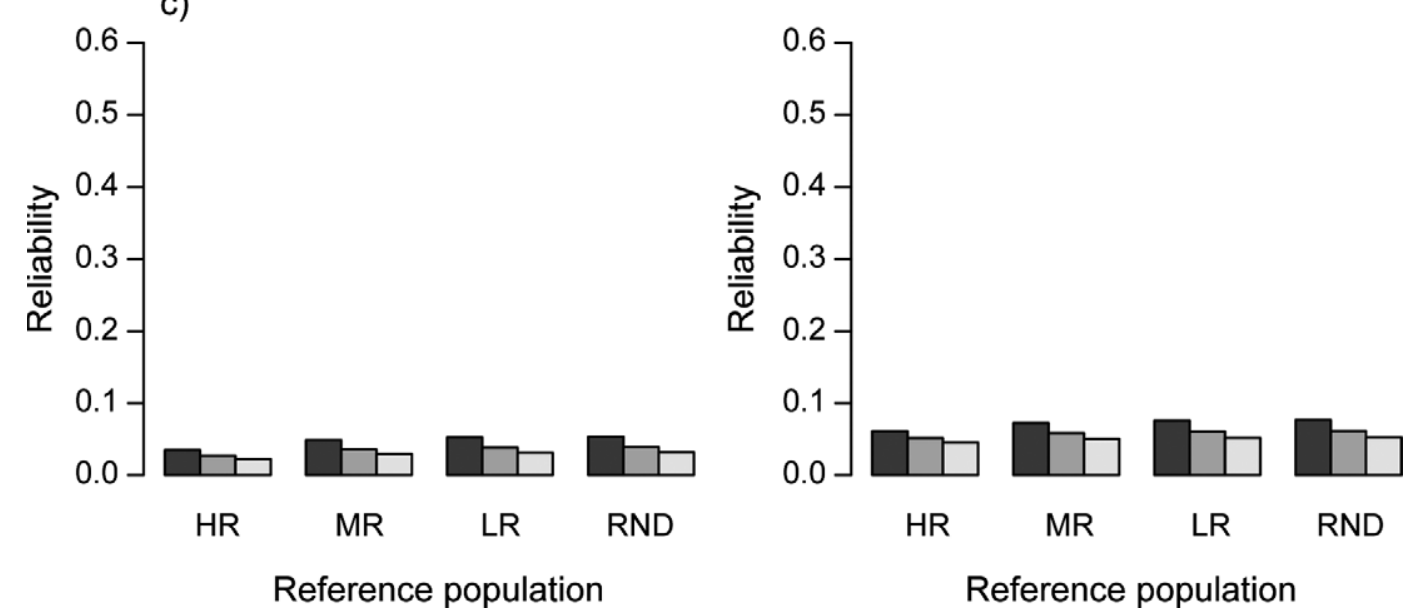

Figure 1. Reliability of breeding values calculated based on pedigree (A) and genomic (B) data across all simulated heritability levels (a: $\mathrm{h}^{2}=0.3 ; \mathrm{b}: \mathrm{h}^{2}=0.05 ; \mathrm{c}: \mathrm{h}^{2}=0.01$ ), 4 reference populations with different family structure (highly related, HR; moderately related, MR; lowly related, LR; random selection of animals, RND), and 3 sets of evaluated animals with different distance from the reference population $(\mathrm{I}=$ the same generation as the animals in the reference population to III $=2$ generations after the reference population) and averaged over all replicates.

\section{DISCUSSION}

This study investigated the effect of relationship of evaluated animals with the reference population and various levels of relationship within the reference population on the reliability of DGV. A small reference population was assumed, consisting of animals with own performance only, to reflect a situation in which the reference population was initiated for scarcely recorded new traits. An important question is whether 


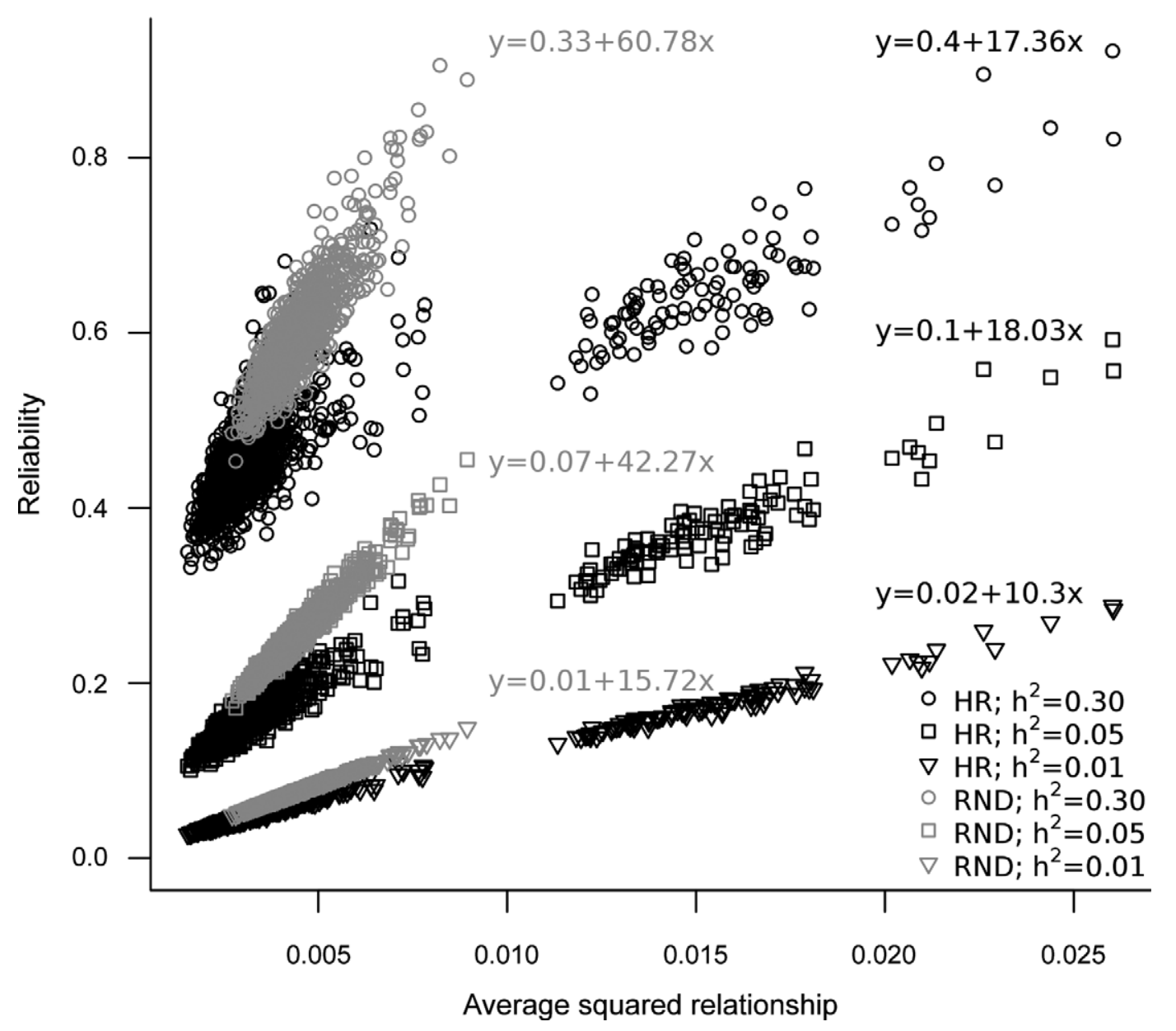

Figure 2. Average squared relationship (based on genomic information) to the reference population versus the reliabilities of genomic breeding values for different heritability levels and the reference populations with high (HR) and low (RND) family structure based on randomly chosen replicate.

our results also apply to large reference populations for traits with abundant phenotypic information available. Additional analyses indicated similar trends when the reference population was twice as large (results not shown). This indicates that optimization of the reference population design, as discussed here, is indeed also applicable to larger reference populations.

\section{Characteristics of the Simulation}

Our initial application of the simulation scheme of de Roos et al. (2009) resulted in a different average LD level than in the study of de Roos et al. (2009) or results from real data sets (obtained with use of the 50K SNP cattle chip). To obtain LD levels as observed in cattle populations (de Roos et al., 2008), the number of mutation events and recombination rates were increased in the first 2 phases of the simulation compared with de Roos et al. (2009). A possible reason for the initial discrepancy with results of de Roos et al.
(2009) is that simulated markers were biallelic in our whole simulation process. In the study of de Roos et al. (2009), however, simulated markers were multiallelic and they were transformed to biallelic at the last stage of the simulation. The genome simulated in this study was smaller than the real genome. This smaller genome size may lead to larger sampling variance for the estimated coefficients in G. Differences between A and G in our study, therefore, might be somewhat different from observed in real data.

\section{Average Relationship in the Reference Population}

The average relationship within the reference population, calculated based on at least 5 generation complete pedigrees and averaged over all replicates for MR (0.0562), LR (0.0497), and RND (0.0487) corresponded to values based on real data (Kearney et al., 2004; König and Simianer, 2006; Mrode et al., 2009). The average relationship within the reference population for 


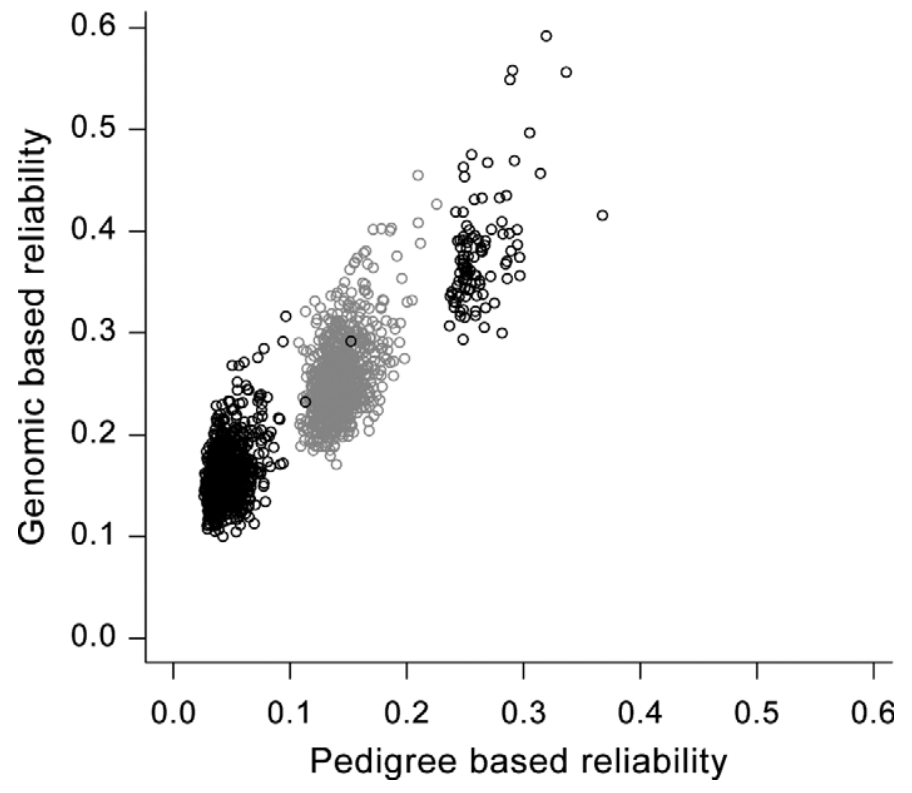

Figure 3. Genomic-based reliability as a function of pedigree-based reliability for high (HR; black) and low (RND; gray) family structure based on a randomly chosen replicate.

HR (0.0946) was higher than the findings of the other studies because of the strong family structure of the population (i.e., all included animals were sired by only 5 individuals; Table 3). The HR population was somewhat similar to a daughter design with large paternal half-sib families that has typically been used in QTL mapping experiments, set up for linkage mapping of QTL (i.e., Weller et al., 1990). The HR gave the low- est DGV reliabilities, which implies that although such experimental designs may be optimal for linkage mapping, they are suboptimal for use in genomic prediction as shown in this study or in genome-wide association studies (Balding, 2006). The RND was a random sample from the simulated population, whereas the other 3 reference populations were constructed by selecting paternal half-sib families of different sizes. Therefore, the family structure of RND was weaker than that in the other 3 reference populations. This weak family structure resulted in a lower average relationship for RND. For example, in RND animals were offspring of 50 sires, whereas in LR animals were offspring of 40 sires.

\section{Reliabilities of Predictions}

The reliabilities in this study were obtained in a similar way to those that can be obtained from the left-hand side of the mixed models equations. For DGV reliabilities obtained in such a way, one of the assumptions is that the markers explain all the genetic variation, whereas some part of the genetic variance is also explained by loci between markers (VanRaden, 2008). This may lead to overestimation of the reliability. Calus et al. (2009), Hayes et al. (2009a), Lund et al. (2009), and Su et al. (2010) also showed that reliabilities obtained from cross-validation are somewhat lower compared with reliabilities calculated from the prediction error variances of the mixed model equations. Therefore, in general, we can expect that reliabil-

Table 4. Reliabilities obtained with use of different sources of information across all reference populations and evaluation sets for heritability of 0.30 averaged over all replicates ${ }^{1}$

\begin{tabular}{|c|c|c|c|c|c|c|}
\hline \multirow[b]{3}{*}{ Scenario $^{2}$} & \multicolumn{6}{|c|}{ Evaluation set ${ }^{3}$} \\
\hline & \multicolumn{2}{|c|}{ I } & \multicolumn{2}{|c|}{ II } & \multicolumn{2}{|c|}{ III } \\
\hline & Mean & SD & Mean & $\mathrm{SD}$ & Mean & $\mathrm{SD}$ \\
\hline \multicolumn{7}{|c|}{ Pedigree data } \\
\hline $\mathrm{HR}$ & 0.121 & 0.001 & 0.079 & 0.004 & 0.054 & 0.002 \\
\hline MR & 0.189 & 0.002 & 0.119 & 0.005 & 0.081 & 0.003 \\
\hline LR & 0.251 & 0.002 & 0.152 & 0.004 & 0.100 & 0.003 \\
\hline RND & 0.274 & 0.001 & 0.165 & 0.002 & 0.107 & 0.002 \\
\hline \multicolumn{7}{|c|}{ Genomic data } \\
\hline $\mathrm{HR}$ & 0.477 & 0.005 & 0.442 & 0.007 & 0.416 & 0.006 \\
\hline MR & 0.542 & 0.004 & 0.490 & 0.007 & 0.457 & 0.006 \\
\hline LR & 0.584 & 0.004 & 0.521 & 0.005 & 0.482 & 0.006 \\
\hline RND & 0.597 & 0.004 & 0.531 & 0.004 & 0.489 & 0.005 \\
\hline
\end{tabular}

${ }^{1}$ Standard deviations of 20 replicates ranged from 0.003 to 0.012 .

${ }^{2} \mathrm{HR}=$ the reference population with the highest average relationship within the reference population; $\mathrm{MR}=$ the reference population with moderate average relationship within the reference population; LR = the reference population with the lowest average relationship within the reference population; RND = the reference population consisted of randomly selected individuals.

${ }^{3}$ Set of evaluation animals chosen from the same generation as the reference animals (I), from offspring of the animals from the same generation as the reference population (II), and from animals 2 generations after the reference population (III). 
a)

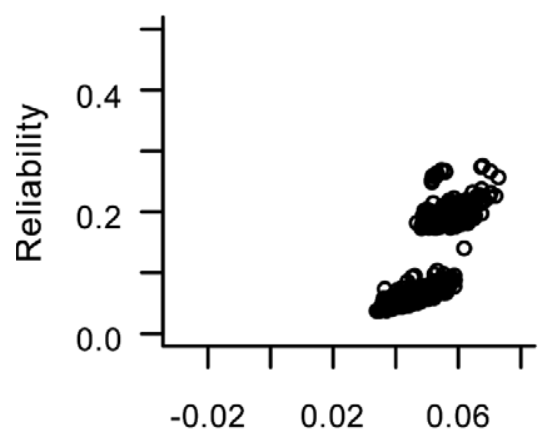

Average relationship

b)

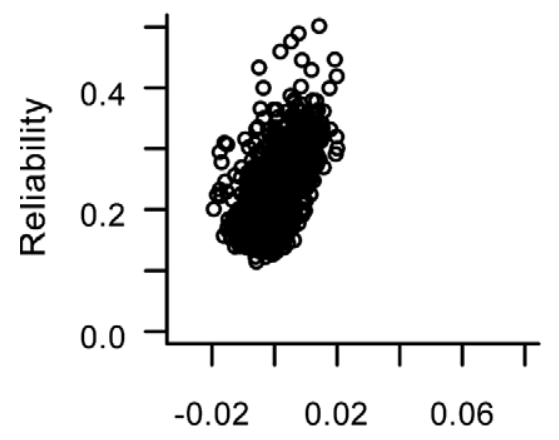

Average relationship

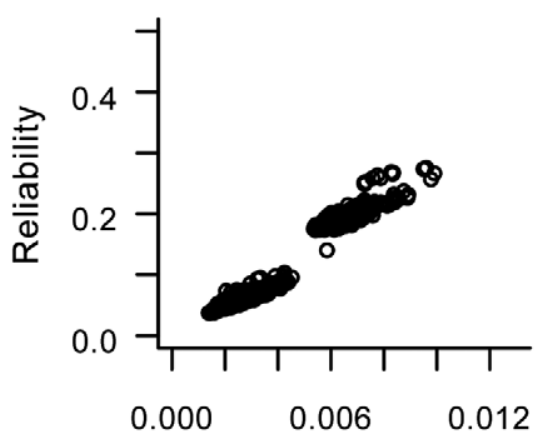

Average squared relationship

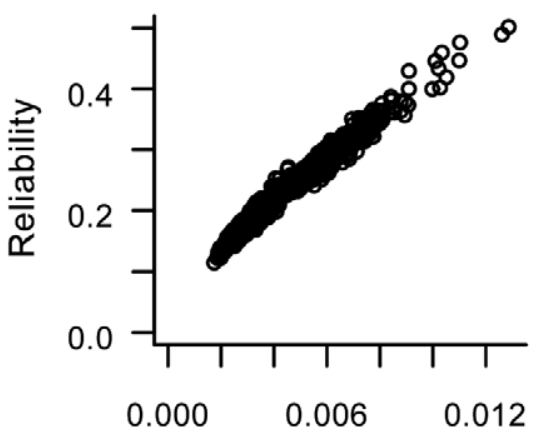

Average squared relationship

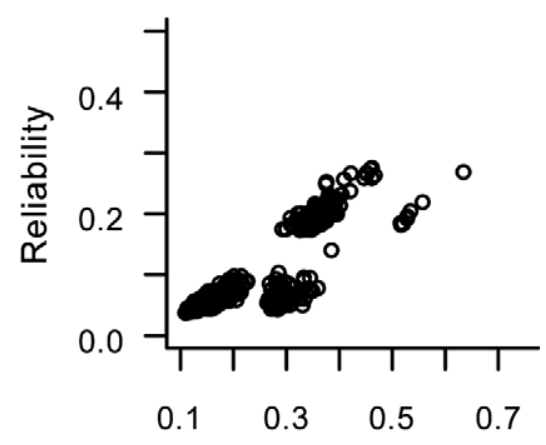

Max. relationship

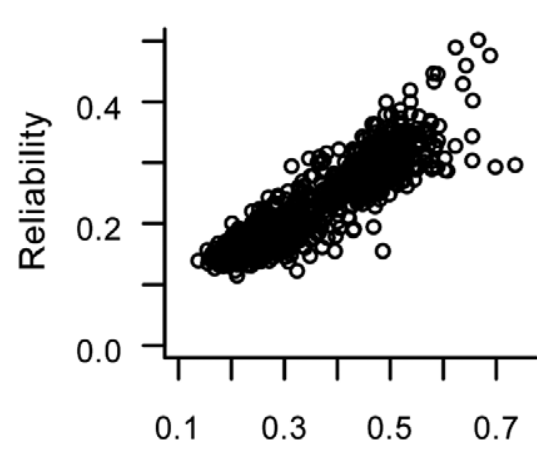

Max. relationship

Figure 4. An illustration of the relationship between 3 different measures of relationship of the evaluated animals to the reliability based on pedigree (a) and genomic (b) data for the reference population with moderate family structure (MR) and heritability of 0.05 , and for the set of evaluated animals originating from the same generation as the reference population (set I).

ities obtained in our study are slightly overestimated. An important question is whether the overestimation of DGV reliabilities is comparable across the different scenarios and reference populations. Reliabilities for the breeding values based on pedigree information were expected to be unbiased. Those gave similar patterns across the reference populations (not shown), despite the level being generally lower. Because replacing the pedigree by genomic data to some extent only adds more information, it is expected that the overestimation of the DGV reliability is mostly a scaling issue.

We also predicted reliabilities deterministically using formulas by Daetwyler et al. (2008) and Goddard (2009) for $N_{e}$ of 100 (Table 5). The reliabilities obtained as in Goddard (2009) were shown to agree with the reliabilities for genomic breeding values in US and Australian Holstein-Friesians and Jerseys (Hayes et al., 2009c). These reliabilities were also in good agreement with the results obtained in the present study. Reliabilities calculated as in Daetwyler et al. (2008) were somewhat higher than those of the current study. Daetwyler et al. (2008) and Goddard (2009) assumed unrelated animals, and therefore, animals with different levels of relationship to the reference population were examined. Examination of animals with the lowest and the highest relationship to the reference population showed that reliabilities predicted for these animals differed from the reliabilities given by the deterministic formulas. Predictions obtained in the present study also allow for assessment of the individual reliability of a particular individual accounting for the actual relationships.

Family Structure of the Reference Population. Comparison between the genomic- and pedigree-based reliabilities presented in Figure 3 shows that 2 groups of evaluated animals can be distinguished for HR. One group, strongly related with the reference population, yielded the highest reliabilities. The other group, loosely related to the reference population, had the lowest reliabilities. Reliabilities for RND was more homogeneous and at an intermediate level. The highest average reliabilities in this study were achieved for the randomly composed reference population (RND). Pérez-Cabal et 
Table 5. Comparison of reliabilities obtained with deterministic formulas of Daetwyler et al. (2008) and Goddard (2009) for all simulated levels of heritability and effective population size $N_{e}$ of 100 with the reliabilities obtained in the present study averaged across all the individuals

\begin{tabular}{lccc}
\hline & \multicolumn{3}{c}{ Reliability } \\
\cline { 2 - 4 } Heritability & $\begin{array}{c}\text { Present } \\
\text { study }\end{array}$ & $\begin{array}{c}\text { Daetwyler et al. } \\
(2008)\end{array}$ & $\begin{array}{c}\text { Goddard } \\
(2009)\end{array}$ \\
\hline 0.30 & 0.55 & 0.75 & 0.59 \\
0.05 & 0.23 & 0.34 & 0.22 \\
0.01 & 0.07 & 0.09 & 0.06 \\
\hline
\end{tabular}

al. (2010) also showed that the highest reliability was obtained when animals were chosen randomly. In their study, unlike our results, RND appeared to have higher average relationship within the reference population than nonrandomly chosen animals. The differences in the average relationship in Pérez-Cabal et al. (2010), however, were small and might be due to sampling; for example, depending on the size of the population from which the reference populations were chosen.

Across different reference populations, and at the same level of the average squared relationship to the reference population, evaluated animals yielded different reliabilities, as shown in Figure 2. The RND population achieved the highest average reliability. The differences, despite the same average squared relationship, were because RND consisted of many small half-sib families and therefore had a low average relationship within the reference population. Related animals may partly explain the same part of variation; therefore, the theoretical maximum reliability can be achieved when all the individuals in the reference population are unrelated and their alleles are not identical-by-state. For such a case, although unrealistic, $\mathbf{A}$ and $\mathbf{G}$ in equations [1] and [2] would be diagonal and the reliability for an evaluated animal would be proportional to its sum of squared relationships to the animals in the reference population, at least when all animals are not inbred. For the reliabilities based on genomic information, according to the suggestion of Calus (2010), animals could be chosen to represent the widest range of possible genotypes to further increase the reliability, which means minimizing the genomic relationships between animals in the reference population, such as with a randomly chosen reference population

Relationship to the Reference Population. The individual reliability strongly depended on the average squared relationship to the reference population (Figure 2 ). This is in accordance with findings of Habier et al. (2010). Variation in the reliability observed at the same level of the average squared relationship could arise, for example, when the animals were similarly related to the reference population but their relatives in the reference population were differentially related to each other.

Larger numbers of generations to the reference population resulted in a decrease in the reliability, as in Habier et al. (2010). The level of genomic reliability decreased more slowly over generations than reliabilities based on pedigree data, as also reported by Wolc et al. (2011). To prevent a decrease of reliability, constant updates of the reference population with animals from more recent generations are required (e.g., animals selected from the evaluation set when their phenotypes become available; Habier et al., 2010).

\section{Implications}

This study showed that an optimally designed reference population should consist of loosely related animals. Still, evaluated animals with low relationship to the reference population had low reliability. In practical applications, for evaluated animals the reliability may be first predicted using pedigree alone. Based on the outcome, a rough estimate of the genomic reliability could be made, and a decision made as to whether the genomic prediction for this animal is reliable enough to justify the genotyping costs. When this is not the case, yet the animal itself is closely related to many other animals that may be evaluated, the animal could be considered for inclusion in the reference population. These aspects require further investigation.

The optimal design of the reference population may differ from one application to another and depend on the desired breeding strategy of the breed. For example, if an experiment intends to measure methane emission, the number of observations is limited because of high phenotyping costs. In such a case, if the goal is to predict breeding values for selection candidates only, then the reference population should consist of animals closely related to these selection candidates. An optimal solution could then be to build the reference population using progeny (i.e., daughters) of key breeding animals (i.e., sires), including only few daughters per sire. If the goal, however, is to predict breeding values (or phenotypes) for all the animals, then a reference population consisting of unrelated animals would be the most desired. Composing the most suitable reference population is therefore an optimization problem because a tradeoff exists between obtaining a low average relationship between the animals in the reference population and a high average squared relationship to the animals in the population to be evaluated.

\section{CONCLUSIONS}

This study shows the importance of optimizing the design of the reference population consisting of cows. 
First, the optimally designed reference population for use in genomic prediction or genome-wide association studies should have a loose family structure; that is, the average relationship within the animals included into the reference population should be low. This implies that different designs are required for reference populations than the traditional designs used for linkage mapping purposes. Second, the relationship between the reference population and the evaluated animal should be maximized. The average squared relationship appeared to be the best measure of the relationship to the reference population with respect to the reliability. Higher levels of heritability resulted in higher levels of reliabilities. To maximize reliability, relationships among animals in the reference population should be minimized and the relationships of the validation animals with the reference population should be maximized

\section{ACKNOWLEDGMENTS}

The authors appreciate useful comments of Johan van Arendonk (Animal Breeding and Genomics Centre, Wageningen University, Wageningen, the Netherlands) and Roel Veerkamp (Animal Breeding and Genomics Centre, Wageningen UR Livestock Research, Lelystad, the Netherlands). Marcin Pszczola gratefully acknowledges the financial support of the Koepon Stichting (Arnhem, the Netherlands), GreenHouseMilk, and Faculty of Animal Breeding and Biology (Poznan University of Life Sciences, Poznan, Poland). The GreenHouseMilk project is financially supported by the European Commission under the Seventh Research Framework Programme, Grant Agreement KBBE-238562. This publication represents the views of the authors, not the European Commission, and the Commission is not liable for any use that may be made of the information.

\section{REFERENCES}

Balding, D. J. 2006. A tutorial on statistical methods for population association studies. Nat. Rev. Genet. 7:781-791.

Berry, D., F. Kearney, and B. Harris. 2009. Genomic selection in Ireland. Pages 29-34 in Proc. Interbull Int. Workshop, Bulletin No. 39. Interbull, Uppsala, Sweden.

Calus, M., H. Mulder, K. Verbyla, and R. Veerkamp. 2009. Estimating reliabilities of genomic breeding values. Pages 198-201 in Proc. Interbull Meeting, Bulletin No. 40, Barcelona, Spain. Interbull, Uppsala, Sweden.

Calus, M. P. L. 2010. Genomic breeding value prediction: Methods and procedures. Animal 4:157-164.

Daetwyler, H. D., B. Villanueva, and J. A. Woolliams. 2008. Accuracy of predicting the genetic risk of disease using a genome-wide approach. PLOS ONE 3:e3395.

de Roos, A. P. W., B. J. Hayes, and M. E. Goddard. 2009. Reliability of genomic predictions across multiple populations. Genetics 183:1545-1553.

de Roos, A. P. W., B. J. Hayes, R. J. Spelman, and M. E. Goddard. 2008. Linkage disequilibrium and persistence of phase in HolsteinFriesian, Jersey and Angus cattle. Genetics 179:1503-1512.
Goddard, M. 2009. Genomic selection: Prediction of accuracy and maximisation of long term response. Genetica 136:245-257.

Goddard, M. E. 2010. Genomic selection in farm animal species-Lessons learnt and future perspectives. Proc. 9th World Congress on Genetics Applied to Livestock Production (WCGALP), Leipzig, Germany. German Society for Animal Science, Neustadt, Germany

González-Recio, O., D. Gianola, N. Long, K. A. Weigel, G. J. M. Rosa, and S. Avendano. 2008. Nonparametric methods for incorporating genomic information into genetic evaluations: An application to mortality in broilers. Genetics 178:2305-2313.

Grapes, L., J. C. M. Dekkers, M. F. Rothschild, and R. L. Fernando. 2004. Comparing linkage disequilibrium-based methods for fine mapping quantitative trait loci. Genetics 166:1561-1570.

Grapes, L., M. Z. Firat, J. C. M. Dekkers, M. F. Rothschild, and R. L. Fernando. 2006. Optimal haplotype structure for linkage disequilibrium-based fine mapping of quantitative trait loci using identity by descent. Genetics 172:1955-1965.

Habier, D., R. L. Fernando, and J. C. M. Dekkers. 2007. The impact of genetic relationship information on genome-assisted breeding values. Genetics 177:2389-2397.

Habier, D., J. Tetens, F.-R. Seefried, P. Lichtner, and G. Thaller. 2010. The impact of genetic relationship information on genomic breeding values in German Holstein cattle. Genet. Sel. Evol. 42:5.

Hayes, B., P. Bowman, A. Chamberlain, K. Verbyla, and M. Goddard. 2009a. Accuracy of genomic breeding values in multi-breed dairy cattle populations. Genet. Sel. Evol. 41:51.

Hayes, B. J., P. J. Bowman, A. J. Chamberlain, and M. E. Goddard. 2009b. Invited review: Genomic selection in dairy cattle: Progress and challenges. J. Dairy Sci. 92:433-443.

Hayes, B. J., H. D. Daetwyler, P. Bowman, G. Moser, B. Tier, R. Crump, M. Khatkar, H. Raadsma, and M. E. Goddard. 2009c. Accuracy of genomic selection: Comparing theory and results. Proc. Assoc. Advmt. Anim. Breed. Genet. 18:34-37.

Heffner, E., M. Sorrells, and J. Jannink. 2009. Genomic selection for crop improvement. Crop Sci. 49:1-12.

Hill, W., and A. Robertson. 1968. Linkage disequilibrium in finite populations. Theor. Appl. Genet. 38:226-231.

Ihara, N., A. Takasuga, K. Mizoshita, H. Takeda, M. Sugimoto, Y. Mizoguchi, T. Hirano, T. Itoh, T. Watanabe, and K. Reed. 2004. A comprehensive genetic map of the cattle genome based on 3802 microsatellites. Genome Res. 14:1987-1998.

Jannink, J.-L., A. J. Lorenz, and H. Iwata. 2010. Genomic selection in plant breeding: From theory to practice. Brief. Funct. Genomics 9:166-177.

Kearney, J. F., E. Wall, B. Villanueva, and M. P. Coffey. 2004. Inbreeding trends and application of optimized selection in the UK Holstein population. J. Dairy Sci. 87:3503-3509.

König, S., and H. Simianer. 2006. Approaches to the management of inbreeding and relationship in the German Holstein dairy cattle population. Livest. Sci. 103:40-53.

Legarra, A., C. Robert-Granie, E. Manfredi, and J.-M. Elsen. 2008. Performance of genomic selection in mice. Genetics 180:611-618.

Lund, M., G. Su, U. Nielsen, and G. Aamand. 2009. Relation between accuracies of genomic predictions and ancestral links to the training data. Pages 162-166 in Proc. Interbull Meeting, Bulletin No. 40, Barcelona, Spain. Interbull, Uppsala, Sweden.

Lund, M. S., A. P. W. de Roos, A. G. de Vries, T. Druet, V. Ducroq, S. Fritz, F. Guillaume, B. Guldbrandtsen, Z. Liu, R. Reents, C. Schrooten, M. Seefried, and G. Su. 2010. Improving genomic prediction by EuroGenomics collaboration. Proc. 9th World Congress on Genetics Applied to Livestock Production (WCGALP), Leipzig, Germany. German Society for Animal Science, Neustadt, Germany.

Meuwissen, T. H. 2009. Accuracy of breeding values of 'unrelated' individuals predicted by dense SNP genotyping. Genet. Sel. Evol. 41:35.

Meuwissen, T. H. E., B. J. Hayes, and M. E. Goddard. 2001. Prediction of total genetic value using genome-wide dense marker maps. Genetics 157:1819-1829.

Mrode, R., J. F. Kearney, S. Biffani, M. Coffey, and F. Canavesi. 2009. Short communication: Genetic relationships between the Holstein 
cow populations of three European dairy countries. J. Dairy Sci. 92:5760-5764.

Pérez-Cabal, M. A., A. I. Vazquez, D. Gianola, G. J. M. Rosa, and K. A. Weigiel. 2010. Accuracy of genomic predictions in USA Holstein cattle from different training-testing designs. Proc. 9th World Congress on Genetics Applied to Livestock Production (WCGALP), Leipzig, Germany. German Society for Animal Science, Neustadt, Germany.

Pszczola, M., H. A. Mulder, and M. P. L. Calus. 2011. Effect of enlarging the reference population with (un)genotyped animals on the accuracy of genomic selection in dairy cattle. J. Dairy Sci. 94:431-441.

Schenkel, F., M. Sargolzaei, G. Kistemaker, G. Jansen, P. Sullivan, B. Van Doormaal, P. VanRaden, and G. Wiggans. 2009. Reliability of genomic evaluation of Holstein cattle in Canada. Pages 51-58 in Proc. Interbull Int. Workshop, Bulletin No. 39. Interbull, Uppsala, Sweden.

Sonesson, A. K., and T. H. Meuwissen. 2009. Testing strategies for genomic selection in aquaculture breeding programs. Genet. Sel. Evol. 41:37.

Su, G., B. Guldbrandtsen, V. R. Gregersen, and M. S. Lund. 2010. Preliminary investigation on reliability of genomic estimated breeding values in the Danish Holstein population. J. Dairy Sci. 93:1175-1183.

VanRaden, P. M. 2008. Efficient methods to compute genomic predictions. J. Dairy Sci. 91:4414-4423.

VanRaden, P. M. C. P. Van Tassell, G. R. Wiggans, T. S. Sonstegard, R. D. Schnabel, J. F. Taylor, and F. S. Schenkel. 2009. Invited review: Reliability of genomic predictions for North American Holstein bulls. J. Dairy Sci. 92:16-24.

Weller, J. I., Y. Kashi, and M. Soller. 1990. Power of daughter and granddaughter designs for determining linkage between marker loci and quantitative trait loci in dairy cattle. J. Dairy Sci. 73:2525-2537.

Wolc, A., J. Arango, P. Settar, J. E. Fulton, N. P. O'Sullivan, R. Preisinger, D. Habier, R. Fernando, D. J. Garrick, and J. C. M. Dekkers. 2011. Persistence of accuracy of genomic estimated breeding values over generations in layer chickens. Genet. Sel. Evol. 43:23.

Yu, K., J. Xu, D. C. Rao, and M. Province. 2005. Using tree-based recursive partitioning methods to group haplotypes for increased power in association studies. Ann. Hum. Genet. 69:577-589.

\section{APPENDIX}

\section{Derivation of Reliability Prediction Equations}

The equations used to predict reliabilities can be derived from selection index theory. Let $\mathbf{P}$ be the (co) variance matrix between the information sources $X_{1}$ to $X_{n}$ and $\mathbf{g}$ be the vector containing the covariances between the information sources and their true breeding values $(\mathbf{A})$. $\mathbf{P}$ contains the single phenotypic observations of genotyped animals in the reference population. Then, $\mathbf{P}$ can be formulated as follows:

$$
\mathbf{P}=\left[\begin{array}{ccc}
\operatorname{var}\left(X_{1}\right) & \ldots & \operatorname{cov}\left(X_{1}, X_{n}\right) \\
\ldots & \ldots & \ldots \\
\operatorname{cov}\left(X_{n}, X_{1}\right) & \ldots & \operatorname{var}\left(X_{n}\right)
\end{array}\right]=\left[\begin{array}{ccc}
\sigma_{P}^{2} & \ldots & a \sigma_{A}^{2} \\
\ldots & \ldots & \ldots \\
a \sigma_{A}^{2} & \ldots & \sigma_{P}^{2}
\end{array}\right],
$$

where $\sigma_{A}^{2}$ is the additive genetic variance, $a$ is the coefficient indicating the proportion of genetic variance shared between the information sources, and $\sigma_{P}^{2}$ is the phenotypic variance. The vector $\mathbf{g}$ can be written as:

$$
\mathbf{g}=\left[\begin{array}{c}
\operatorname{cov}\left(A, X_{1}\right) \\
\ldots \\
\operatorname{cov}\left(A, X_{n}\right)
\end{array}\right]=\left[\begin{array}{c}
a \sigma_{A}^{2} \\
\cdots \\
a \sigma_{A}^{2}
\end{array}\right] .
$$

Then, the reliability of breeding values estimated with use of selection index theory can be calculated as $r_{I H}^{2}=\frac{\mathbf{b}^{\prime} \mathbf{g}}{\sigma_{A}^{2}}$, where $\mathbf{b}$ is a vector with optimal weights for the information sources calculated as $\mathbf{b}=\mathbf{P}^{-1} \mathbf{g}$. Therefore, the equation for the reliability can be rewritten as

$$
r_{I H}^{2}=\frac{\mathbf{g}^{\prime} \mathbf{P}^{-1} \mathbf{g}}{\sigma_{A}^{2}} .
$$

Let $\mathbf{A}$ be the additive relationship matrix formulated as

$$
\mathbf{A}=\left[\begin{array}{ccc}
a_{11} & \ldots & a_{1 n} \\
\ldots & \ldots & \ldots \\
a_{n 1} & \ldots & a_{n n}
\end{array}\right]
$$

and $\sigma_{A}^{2}$ equals 1 , then $\mathbf{P}$ can be rewritten as $\left[\mathbf{A}+\mathbf{I}\left(\frac{\sigma_{e}^{2}}{\sigma_{A}^{2}}\right)\right]$, where variance ratio $\left(\frac{\sigma_{e}^{2}}{\sigma_{A}^{2}}\right)$ reflects the heritability, because $\frac{\sigma_{e}^{2}}{\sigma_{A}^{2}}=\frac{1}{h^{2}}-1$, and $\mathbf{A}$ is created for the information sources (i.e., reference population); then, $\mathrm{g}$ will equal the additive relationship of the evaluated animal to the reference population a. Therefore, reliability of traditional selection can be calculated as $r_{A}^{2}=\mathbf{a}\left[\mathbf{A}+\mathbf{I}\left(\frac{\sigma_{e}^{2}}{\sigma_{A}^{2}}\right)\right]^{-1} \mathbf{a}^{\prime}$. For genomic selection, pedigreebased coefficients can be substituted by the genomic coefficients as $r_{G}^{2}=\mathbf{c}\left[\mathbf{G}+\mathbf{I}\left(\frac{\sigma_{e}^{2}}{\sigma_{A}^{2}}\right)\right]^{-1} \mathbf{c}^{\prime}$, where $\mathbf{G}$ is genomic relationship matrix for the animals in the reference population and $\mathbf{c}$ is the genomic-based relationship of the evaluated animal to the reference population. 